\title{
Effective Packet Scheduling with Fairness Adaptation in Ultra-Wideband Wireless Networks
}

\author{
Hai Jiang, Student Member, IEEE, and Weihua Zhuang, Senior Member, IEEE
}

\begin{abstract}
Ultra-wideband (UWB) transmission is an emerging wireless technology, and medium access control (MAC) with quality of service $(\mathrm{QoS})$ provisioning is essential to coordinate the access among competing devices in UWB-based wireless networks. In this paper, we study the exclusive region concept (which was previously proposed) to determine the active set of senders at a time. We find out that, different from the previous work, the exclusive region for a specific link should be a systemlevel concept, and should depend on system factors such as interference from/to other active links. Based on the findings, two MAC packet scheduling schemes are proposed to exploit the system capacity and, at the same time, to achieve a certain level of fairness in UWB wireless networks. As the long acquisition time in UWB transmission can significantly reduce the system efficiency, the proposed schemes can be modified to alleviate the negative effect of a long acquisition time. Computer simulations demonstrate the effectiveness and efficiency of our proposed schemes.
\end{abstract}

Index Terms - Ultra-wideband (UWB) transmission, medium access control (MAC), packet scheduling, quality of service (QoS), transmission power and rate, fairness.

\section{INTRODUCTION}

$\mathbf{U}$ LTRA-WIDEBAND (UWB) transmission is an emerging wireless technology, and has been considered as one of the promising technologies to provide multimedia services in both indoor and outdoor applications. A UWB system is defined as any radio system that has the $-10 \mathrm{~dB}$ fractional bandwidth (or $-10 \mathrm{~dB}$ bandwidth) more than $20 \%$ (or 500 $\mathrm{MHz}$ ). With the unique merits such as high rate, low power spectral density, capability to capture multipath energy, and ability of accurate positioning, UWB has demonstrated its potential in future multimedia applications as well as in industrial control and maintenance, medical monitoring, radar imaging, home automation, Department of Defense (DoD) systems, etc. As a significant breakthrough for R\&D on UWB, the Federal Communications Commission (FCC) has allowed UWB indoor applications in the frequency band from 3.1 to 10.6 GHz on an unlicensed basis [1].

In a UWB network, the wireless medium is shared among mobile nodes. To achieve desired quality of service (QoS)

Manuscript received May 10, 2005; revised November 23, 2005 and February 9, 2006; accepted February 13, 2006. The associate editor coordinating the review of this paper and approving it for publication was V. Leung. This work was supported by the Premier's Research Excellence Award (PREA) from the Ontario Government, and a research grant from the Natural Science and Engineering Research Council (NSERC) of Canada.

H. Jiang is with the Electrical Engineering Department, Princeton University, Princeton, New Jersey 08544 USA (email: haijiang@ princeton.edu).

W. Zhuang is with the Department of Electrical and Computer Engineering, University of Waterloo, 200 University Avenue West, Waterloo, Ontario, Canada N2L 3G1 (e-mail: wzhuang@bbcr.uwaterloo.ca).

Digital Object Identifier 10.1109/TWC.2007.05340. (e.g., in terms of transmission accuracy, delay/jitter, throughput, fairness), the multiple access to the channel should be coordinated by a medium access control (MAC) mechanism in an effective and orderly manner. For traditional wireless local area networks (WLANs) or ad hoc networks, many MAC protocols have been developed, such as ALOHA and its slotted version, and carrier sense multiple access (CSMA)based random access protocols. They are all contention-based with a single channel (thereby termed single-channel case), and hence two nearby simultaneous transmissions may collide. However, the inherent spread spectrum in UWB can support simultaneous transmissions, with an appropriate pseudorandom sequence design and effective call admission control (CAC), referred to as multi-channel case. In the multi-channel case, two nearby transmissions do not collide, but rather generate interference to each other, thus requiring different coordination mechanisms from those in the single-channel case. In the literature, one major stream of UWB MAC research is IEEE 802.15.3, which is designed for short-range ad hoc connectivity in wireless personal area networks (WPANs). However, it is not explicitly designed for the UWB-based multi-channel transmissions.

For multi-channel multiple access, in the limit of infinite bandwidth $(W \rightarrow \infty)$, the optimal MAC scheme is to simply allow transmissions over all the links simultaneously, because interference becomes negligible [2]. However, for a practical UWB network, the bandwidth is large but finite, so that uncontrolled simultaneous transmissions are not optimal [3], [4]. Hence, it is critical to determine when, where, and how to allow simultaneous transmissions, and how to alleviate the induced interference in order to achieve desired performance. This research is to contribute to the development of such packet scheduling schemes in UWB wireless networks.

The contribution of this paper is two-fold: 1) we demonstrate that the exclusive region concept previously proposed in the literature is not optimal in terms of throughput; 2) we propose sub-optimal (in terms of throughput) packet scheduling schemes to achieve fairness and at the same time alleviate the effect of long acquisition overhead in UWB multi-channel transmissions.

The rest of this paper is organized as follows. In Section II, the system model is described. Section III reviews the concept of exclusive region in previous research work and presents our studies. In Section IV, we propose two MAC scheduling schemes to efficiently utilize the bandwidth, achieve a certain level of fairness among wireless links, and alleviate the effect of a long acquisition time on UWB transmissions. Section V is devoted to performance evaluation of the proposed schemes, followed by concluding remarks in Section VI. 


\begin{tabular}{|c|c|c|c|c|}
\hline Beacon & $\begin{array}{c}\text { Control } \\
\text { slot }\end{array}$ & $\begin{array}{c}\text { Packet } \\
\text { slot }\end{array}$ & $\cdots$ & $\begin{array}{c}\text { Packet } \\
\text { slot }\end{array}$ \\
\hline
\end{tabular}

Fig. 1. Frame structure in the UWB wireless network.

\section{SySTEM MODEL}

\section{A. Frame Architecture}

We consider a UWB wireless network with a number of nodes which communicate with each other on a peer-topeer basis. The nodes are with low mobility. Among the nodes, one is selected as the central controller. The main responsibility of the central controller is to provide timing and global information, and perform resource allocation for the active connections in the UWB network. In general, the central controller can be a node with sufficient power supply and advantageous location. The central controller can change from time to time for load distribution, or due to node arrivals/departures and user mobility.

At the physical layer, the implementation of a UWB transmission can be achieved by pulse-based time-hopping (TH) or pulse-based direct sequence (DS), or multiband orthogonal frequency division multiplexing (MB-OFDM). In the following, we use TH-UWB as an example. However, the principles can be extended to DS-UWB and MB-OFDM systems.

In the UWB network, time is partitioned into frames, each of which is further divided into a beacon, a control slot, and a number of packet slots, as shown in Fig. 1. A guard time exists between any two slots, which is not shown in the figure. The beacon is used by the central controller to provide timing and global information (such as the time hopping sequences used for channel request), and broadcast scheduling decision for the packet slots. When a node has a call arrival, it selects one of the time hopping sequences indicated in the beacon, and sends a request via this sequence in the control slot. The request is per-call based, thus having a limited overhead. The central controller monitors all the channels associated with the announced time hopping sequences. Upon correct reception of one request, in the subsequent beacon, the central controller announces whether or not the request can be admitted, and if yes, at which slots and with what power and rate levels. In other words, the central controller is responsible for an effective and efficient resource allocation with QoS provisioning in each packet slot, which is the focus of this paper. For each link, the transmission at the packet slots is via a time hopping sequence private to the sourcedestination pair [5].

\section{B. Channel Model}

The rich resolvable multipath components in pulse-based UWB networks determine that UWB signal reception does not suffer much from multipath fading [6]-[8]. Thus, similar to [4], we assume that there is no fast fading, and the power at the receiver is attenuated only due to path loss, i.e., the channel gain from link $i$ 's transmitter to link $j$ 's receiver can be represented as

$$
h_{i j}=K \cdot d_{i j}^{-\theta}
$$

where $K$ and $\theta$ are constants, and $d_{i j}$ is the distance from link $i$ 's transmitter to link $j$ 's receiver.

Since UWB systems exhibit unique physical layer characteristics such as precise positioning capability, the central controller can know the location information of all the nodes (with low mobility) [9], thus has the channel gain information of all links when multipath fading can be addressed by the RAKE receiver.

\section{QoS Model}

In TH-UWB, the information bit is transmitted with a train of very narrow pulses (usually in the order of a nanosecond). Multiple access in TH-UWB can be achieved by assigning unique time hopping sequences to different links. Simultaneous transmissions do not collide, but rather generate interference to each other. It has been shown in [10] that the total interference from a large number of links can be approximated as Gaussian noise. Based on this approximation, for a $\mathrm{TH}-$ UWB network with $N$ active links, the achieved signal to interference-plus-noise ratio (SINR) at the receiver of link $i$ can be represented as

$$
\operatorname{SINR}_{i}=\frac{P_{i} h_{i i}}{R_{i}\left(\eta_{i}+T_{f} \sigma^{2} \sum_{j=1, j \neq i}^{N} P_{j} h_{j i}\right)}, \quad i=1, \ldots, N
$$

where $P_{i}$ denotes the average transmission power of link $i$ 's transmitter, $R_{i}$ bit rate of link $i, \eta_{i}$ the background noise energy plus interference from other non-UWB systems, $T_{f}$ the pulse repetition time, and $\sigma^{2}$ a parameter depending on the shape of the pulse [11].

QoS in MAC can be classified according to its implementation in UWB networks, based on a hierarchy of two different levels: bit-level and packet-level. Bit-level QoS is to ensure some degree of transmission accuracy, normally represented by an upper bound on bit error rate (BER). The BER guarantee can be achieved by satisfying a required SINR value $\gamma_{i}$ for link $i$. For UWB transmission, the one-to-one mapping of BER to SINR depends on channel characteristics, modulation, channel coding, diversity, and receiver design. On the other hand, transmission rate (i.e., throughput), timeliness (i.e., delay and jitter), and fairness are the main consideration in packet-level QoS. In this research, the objective in QoS provisioning is to maximize system throughput with a certain level of fairness, under the constraint of the required SINR bound. That is, for each link $i$, the following inequality should hold

$$
\frac{P_{i} h_{i i}}{R_{i}\left(\eta_{i}+T_{f} \sigma^{2} \sum_{j=1, j \neq i}^{N} P_{j} h_{j i}\right)} \geq \gamma_{i} .
$$

\section{Full Power Transmission}

An equivalent form of inequality (3) is

$$
R_{i} \leq \frac{P_{i} h_{i i}}{\gamma_{i}\left(\eta_{i}+T_{f} \sigma^{2} \sum_{j=1, j \neq i}^{N} P_{j} h_{j i}\right)} .
$$

The inequality gives the maximum achievable bit rate of link $i$ with the constraint of SINR value $\gamma_{i}$. This is under the assumption that adaptive rate can be achieved by changing the processing gain, e.g., via adapting the number of pulses for each symbol and/or maximum time hopping shift, or using 


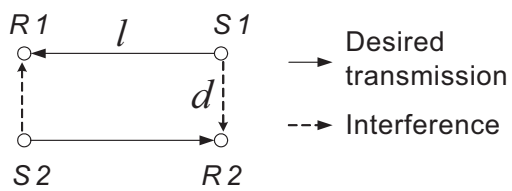

Fig. 2. The near-far scenario [5].

adaptive channel coding such as rate compatible punctured convolutional (RCPC) code [5]. To achieve link adaptation, a feedback channel is necessary. In our system, a receiver can send the feedback with low power and low rate in the control slot to the central controller or in a packet slot to its sender. For the simplicity of analysis, we omit the effect of the feedback channel. On the other hand, for each link, the maximum achieved throughput should not exceed $1 / T_{f}$ as the processing gain should be at least 1 . Hence, for link $i$ the achievable rate is

$$
\min \left\{\frac{1}{T_{f}}, \frac{P_{i} h_{i i}}{\gamma_{i}\left(\eta_{i}+T_{f} \sigma^{2} \sum_{j=1, j \neq i}^{N} P_{j} h_{j i}\right)}\right\} .
$$

For simplicity of presentation, in the following, when a rate appears in a format similar to $\frac{P_{i} h_{i i}}{\gamma_{i}\left(\eta_{i}+T_{f} \sigma^{2} \sum_{j=1, j \neq i}^{N} P_{j} h_{j i}\right)}$, it actually has an attached condition of the upper bound value $1 / T_{f}$.

To maintain desired transmission quality, the traditional way is to apply power control at each link to achieve the desired SINR. However, recent research [4], [12] has shown that, for ad hoc connectivity, the optimal MAC layer should make use of the allowed maximum power at each active link, and that power control does not provide a significant gain when dynamic channel coding is used. Hence, in our research, each transmission is with the maximum allowed power, which can be determined by the emission regulation and the energy consumption of the terminals. This means that, at any moment, each sender either transmits with maximum allowed power, or does not transmit at all. At a specific packet slot, it is critical to determine the set of active links with full power transmission while the others keep silent. To address this issue, in the literature, an exclusive region concept was proposed [4], [5].

\section{EXCLUSIVE REGION CONCEPT}

\section{A. Review of the Exclusive Region Concept}

UWB transmission is generally characterized by low power and low interference. If two transmission links are separated by a large distance, the interference between them may be negligible. Thus, it is optimal (in terms of throughput) to allow the two links to transmit simultaneously. The exclusive region concept is one effort to define such a "large distance". When a specific link is transmitting, the concept allows simultaneous transmissions from interfering sources outside the exclusive region, while senders inside the exclusive region are required to keep silent. This means senders inside the exclusive region operate in a time-division multiple access (TDMA) mode.

To determine the exclusive region size (i.e., the minimum distance $d$ from the interfering source to a desired receiver), in [4] $d$ is selected so as to maximize the achieved rate at the desired receiver. However, rate maximization of a specific link may not lead to the maximization of the overall system throughput. In [5], a near-far scenario is used to determine $d$. As shown in Fig. 2, two sources $S 1$ and $S 2$ are intended to transmit to two destinations $R 1$ and $R 2$, respectively. The length of both links is $l$, while the distance from an interferer to the desired receiver is $d$. Two scheduling schemes are investigated: All-at-Once allows both links to send all the time, while TDMA permits only one link at a time, and the duty cycle of each link is $50 \%$. Each link uses full maximum power when transmitting. The exclusive region size $d$ is chosen as the point where TDMA performs equally with the All-atOnce scheme in terms of rate achieved by source $S 1$. Indeed, this method is effective if there exist only two links in the neighborhood. However, if other UWB links exist, from the two possible transmissions $S 1 \rightarrow R 1$ and $S 2 \rightarrow R 2$, other links experience interference of one full power transmission in TDMA, but two full power transmissions in All-at-Once. Taking into account the difference in the total transmitted power levels, it can be concluded that the exclusive region size should be larger than that determined based on the method in [5]. In addition, it is not necessary to let the two links evenly share the time in the TDMA mode.

\section{B. Discussion}

Consider two target transmission links $i$ and $j$ in a UWB wireless network. Denote the set of other active links as $\mathcal{S}$. We also investigate two scenarios with respect to the two target links:

- All-at-Once: both links $i$ and $j$ transmit all the time with full maximum power.

- TDMA: links $i$ and $j$ transmit one by one based on round robin, with duty cycles $\phi_{i}$ and $\phi_{j}\left(=1-\phi_{i}\right)$ respectively.

In both scenarios, the set $\mathcal{S}$ is the same, and the processing gain and/or channel coding is adapted to the experienced interference at the receiver in order to get the maximum achievable rate.

In All-at-Once, the system throughput can be calculated as

$$
\begin{gathered}
T^{\mathrm{All}}=\frac{P_{i} h_{i i}}{\gamma_{i}\left[\eta_{i}+T_{f} \sigma^{2}\left(\sum_{k \in \mathcal{S}} P_{k} h_{k i}+P_{j} h_{j i}\right)\right]} \\
+\frac{P_{j} h_{j j}}{\gamma_{j}\left[\eta_{j}+T_{f} \sigma^{2}\left(\sum_{k \in \mathcal{S}} P_{k} h_{k j}+P_{i} h_{i j}\right)\right]} \\
+\sum_{k \in \mathcal{S}} \frac{P_{k} h_{k k}}{\gamma_{k}\left[\eta_{k}+T_{f} \sigma^{2}\left(\sum_{n \in \mathcal{S}, n \neq k} P_{n} h_{n k}+P_{i} h_{i k}+P_{j} h_{j k}\right)\right]} .
\end{gathered}
$$

On the right side of the equation, the first and the second terms are the achieved rates of links $i$ and $j$, respectively, and the last term is the sum of achieved rates of all other active links.

In TDMA, when link $i$ is active, the system throughput is

$$
\begin{aligned}
& T_{i}^{\mathrm{TDMA}}=\frac{P_{i} h_{i i}}{\gamma_{i}\left[\eta_{i}+T_{f} \sigma^{2} \sum_{k \in \mathcal{S}} P_{k} h_{k i}\right]} \\
& \quad+\sum_{k \in \mathcal{S}} \frac{P_{k} h_{k k}}{\gamma_{k}\left[\eta_{k}+T_{f} \sigma^{2}\left(\sum_{n \in \mathcal{S}, n \neq k} P_{n} h_{n k}+P_{i} h_{i k}\right)\right]}
\end{aligned}
$$


and when link $j$ is active, the system throughput is

$$
\begin{aligned}
& T_{j}^{\mathrm{TDMA}}=\frac{P_{j} h_{j j}}{\gamma_{j}\left[\eta_{j}+T_{f} \sigma^{2} \sum_{k \in \mathcal{S}} P_{k} h_{k j}\right]} \\
& \quad+\sum_{k \in \mathcal{S}} \frac{P_{k} h_{k k}}{\gamma_{k}\left[\eta_{k}+T_{f} \sigma^{2}\left(\sum_{n \in \mathcal{S}, n \neq k} P_{n} h_{n k}+P_{j} h_{j k}\right)\right]} .
\end{aligned}
$$

Therefore, it can be seen that TDMA is more advantageous than All-at-Once if there exists a $\phi_{i} \in[0,1]$ that satisfies

$$
\phi_{i} T_{i}^{\mathrm{TDMA}}+\left(1-\phi_{i}\right) T_{j}^{\mathrm{TDMA}}>T^{\mathrm{All}} .
$$

Without loss of generality, we assume $T_{i}^{\mathrm{TDMA}} \geq T_{j}^{\mathrm{TDMA}}$. Then (8) is equivalent to

$$
\phi_{i}>\frac{T^{\mathrm{All}}-T_{j}^{\mathrm{TDMA}}}{T_{i}^{\mathrm{TDMA}}-T_{j}^{\mathrm{TDMA}}} .
$$

Taking into account $\phi_{i} \in[0,1]$, it can be concluded that there exists a $\phi_{i} \in[0,1]$ that satisfies (9) if

$$
T_{i}^{\mathrm{TDMA}}>T^{\mathrm{All}}
$$

or equivalently if $\max \left\{T_{i}^{\mathrm{TDMA}}, T_{j}^{\mathrm{TDMA}}\right\}>T^{\mathrm{All}}$ in general.

It can be seen that, for the two target links, it is not feasible to tell whether or not TDMA is better than All-at-Once based only on interference between them (as in the case of [5]). In contrast, as all active links in UWB wireless networks interfere with each other, a system-level consideration should be taken to determine whether or not a link should transmit when another link is transmitting. This means the exclusive region of a specific link should be a system-level concept. More specifically, the interference from/to other existing active links plays an important role in determining whether the two target links should transmit simultaneously or one by one.

\section{Proposed MAC Scheduling Schemes}

Consider $N$ links in $L$ packet slots. For packet slot $l \in$ $\{1, \ldots, L\}, \mathbf{r}^{l}=\left\{r_{1}^{l}, \ldots, r_{N}^{l}\right\} \in \mathbb{R}^{N}$ denotes the transmission rate vector, and $\boldsymbol{\alpha}^{l}=\left\{\alpha_{1}^{l}, \ldots, \alpha_{N}^{l}\right\} \in\{0,1\}^{N}$ is the link activity (" 1 " means that the link is active with maximum allowed transmission power, and "0" means that the link keeps silent). Hence, an optimization problem can be formulated as follows

$$
\begin{aligned}
& \underset{\left\{\boldsymbol{\alpha}^{1}, \ldots, \boldsymbol{\alpha}^{L}\right\}}{\operatorname{Maximize}} \quad \sum_{l=1}^{L} \sum_{n=1}^{N} r_{n}^{l} \\
& \text { where } \quad r_{n}^{l}=\frac{\alpha_{n}^{l} P_{n} h_{n n}}{\gamma_{n}\left(\eta_{n}+T_{f} \sigma^{2} \sum_{j \neq n} \alpha_{j}^{l} P_{j} h_{j n}\right)} .
\end{aligned}
$$

High complexity is expected to solve the optimization problem. In addition, at the optimal point, it is possible that some links may starve. Hence, we propose sub-optimal approaches, based on the discussion given in the previous section, beginning with a utility function definition.

\section{A. Utility Function}

Recall that if $T_{i}^{\mathrm{TDMA}} \geq T_{j}^{\mathrm{TDMA}}$ and $T_{i}^{\mathrm{TDMA}}>T^{\mathrm{All}}$, it is better (in terms of system throughput) to let links $i$ and $j$ transmit by TDMA scheduling, with link $i$ having duty cycle $\phi_{i}$ more than $\max \left\{0,\left(T^{\mathrm{All}}-T_{j}^{\mathrm{TDMA}}\right) /\left(T_{i}^{\mathrm{TDMA}}-T_{j}^{\mathrm{TDMA}}\right)\right\}$. Apparently, if we set $\phi_{i}=1$, the system throughput achieves the maximum value, at the cost of the starvation of link $j$. In the following, we first show how this principle can be used in the scheduling of a UWB network to achieve maximum system throughput, then we try to take into account the fairness issue.

After some mathematical manipulation, TDMA condition (10) can be rewritten as (12) at the top of next page. By defining $\mathcal{S}^{\prime}=\mathcal{S} \cup\{i\}$ (i.e., let link $i$ be active), the inequality can be further rewritten as (13). On the other hand, for the active link set $\mathcal{S}^{\prime}$ at a packet slot, if link $j$ is also active at the packet slot, the gain obtained by link $j$ is defined as its achieved rate, calculated by

$$
G_{j j}=\frac{P_{j} h_{j j}}{\gamma_{j}\left(\eta_{j}+T_{f} \sigma^{2} \sum_{k \in \mathcal{S}^{\prime}} P_{k} h_{k j}\right)}
$$

and the cost (due to the increased interference by link $j$ ) to a link $k \in \mathcal{S}^{\prime}$ is defined as the reduction of link $k$ 's achieved rate, given by

$$
\begin{aligned}
C_{j k} & =\frac{P_{k} h_{k k}}{\gamma_{k}\left[\eta_{k}+T_{f} \sigma^{2} \sum_{n \in \mathcal{S}^{\prime}, n \neq k} P_{n} h_{n k}\right]} \\
& -\frac{P_{k} h_{k k}}{\gamma_{k}\left[\eta_{k}+T_{f} \sigma^{2}\left(\sum_{n \in \mathcal{S}^{\prime}, n \neq k} P_{n} h_{n k}+P_{j} h_{j k}\right)\right]} .
\end{aligned}
$$

A utility function is defined to represent the "net gain" of link $j$ being active in a packet slot:

$$
U_{j}=G_{j j}-\sum_{k \in \mathcal{S}^{\prime}} C_{j k} .
$$

Note that the value of the utility function depends on the set $\mathcal{S}^{\prime}$. For the same link $j$ and packet slot, the utility may be positive for an $\mathcal{S}^{\prime}$ set and negative for another.

Thus, we have an equivalent form of the condition (13) for TDMA scheduling:

$$
G_{j j}-\sum_{k \in \mathcal{S}^{\prime}} C_{j k}<0
$$

Intuitively, at a packet slot, if the "net gain" of link $j$ being active is negative, we should keep link $j$ silent in order to achieve a larger system throughput. Based on this principle, we propose two MAC packet scheduling schemes for each packet slot, termed water-draining and water-adding, respectively.

\section{B. Water-Draining and Water-Adding Packet Scheduling}

The basic idea of water-draining is to first assume all the communication links are active, then remove the link (from the active set denoted by $\mathcal{S})^{1}$ having a negative utility function with the maximum magnitude. This procedure is repeated until all the remaining active links are with positive utility values. On the other hand, the water-adding first assumes all the links are idle. It then randomly chooses a link to be active and, among the remaining idle links, activates the one with the largest positive utility value. This procedure is repeated until all the remaining idle links have negative utility values.

By either of the procedures, the capacity in a packet slot is maximally exploited. Apparently, if all packet slots follow the same procedure, with low mobility, some links in advantageous positions (such as with a short link distance or in a low-interference neighborhood) may obtain excessive

\footnotetext{
${ }^{1}$ For simplicity of presentation, we omit the slot index of $\mathcal{S}$ and other symbols in the schemes.
} 


$$
\begin{aligned}
& \frac{P_{j} h_{j j}}{\gamma_{j}\left[\eta_{j}+T_{f} \sigma^{2}\left(\sum_{k \in \mathcal{S}} P_{k} h_{k j}+P_{i} h_{i j}\right)\right]}-\left\{\frac{P_{i} h_{i i}}{\gamma_{i}\left[\eta_{i}+T_{f} \sigma^{2} \sum_{k \in \mathcal{S}} P_{k} h_{k i}\right]}-\frac{P_{i} h_{i i}}{\gamma_{i}\left[\eta_{i}+T_{f} \sigma^{2}\left(\sum_{k \in \mathcal{S}} P_{k} h_{k i}+P_{j} h_{j i}\right)\right]}\right\} \\
& -\left\{\sum_{k \in \mathcal{S}} \frac{P_{k} h_{k k}}{\gamma_{k}\left[\eta_{k}+T_{f} \sigma^{2}\left(\sum_{n \in \mathcal{S}, n \neq k} P_{n} h_{n k}+P_{i} h_{i k}\right)\right]}-\sum_{k \in \mathcal{S}} \frac{P_{k}\left[\eta_{k}+T_{f} \sigma^{2}\left(\sum_{n \in \mathcal{S}, n \neq k} P_{n} h_{n k}+P_{i} h_{i k}+P_{j} h_{j k}\right)\right]}{\gamma_{k} h_{k k}}<0 . \quad\right. \text { (12) } \\
& \frac{P_{j} h_{j j}}{\gamma_{j}\left(\eta_{j}+T_{f} \sigma^{2} \sum_{k \in \mathcal{S}^{\prime}} P_{k} h_{k j}\right)}-\sum_{k \in \mathcal{S}^{\prime}}\left\{\frac{P_{k} h_{k k}}{\gamma_{k}\left[\eta_{k}+T_{f} \sigma^{2} \sum_{n \in \mathcal{S}^{\prime}, n \neq k} P_{n} h_{n k}\right]}-\frac{T_{k}\left[\eta_{k}+T_{f} \sigma^{2}\left(\sum_{n \in \mathcal{S}^{\prime}, n \neq k} P_{n} h_{n k}+P_{j} h_{j k}\right)\right]}{\gamma_{k}}\right\} 0 .
\end{aligned}
$$

services while others may starve. It is desired to address a certain level of fairness while at the same time exploiting the wireless capacity.

\section{Fairness Adaptation}

For a wireless network, the system throughput can be increased if at any instant resources are allocated to users with a good channel quality, at the cost of possible service starvation of users with a poor channel. Hence, efforts are needed to make a good compromise between the throughput and fairness. For a UWB wireless network, to achieve fairness is technically very challenging. First, the notion of fairness is quite different from that in traditional wireline networks or packet cellular networks, where fairness can be defined for a specific link with a fixed capacity. In a UWB wireless network, the links interfere with each other. The interfering relationship among links in a large area determines that the fairness in UWB should have a global definition instead of being limited to a specific link. Second, spatial channel reuse may conflict with fairness. As fairness is a global notation, it requires that the sources transmit based on a specific order. On the other hand, to take advantage of spatial channel reuse, two links with a large space separation can transmit simultaneously, which may violate the link transmission order determined by the strict fairness. Hence, a feasible tradeoff should be considered [13]. The well-know proportional fair scheduling obtains a good compromise between throughput and fairness for codedivision multiple access (CDMA) cellular systems. For each time slot, the scheduler schedules the user with the highest priority value defined as the ratio of SINR to the average throughput of the user in a certain time window [14]. If a user has a low average throughput, its chance to be selected to transmit is relatively large, so as to achieve a certain level of fairness. This principle is designed for the case when only one user is scheduled at any time, thus not being able to be applied directly to wireless networks with multiple transmissions.

Here we introduce a method for a UWB wireless network to achieve a good tradeoff between system throughput and fairness. The basic idea is that users with good channel quality $^{2}$ are given priority while at the same time users with poor channel quality get an acceptable service share. Specifically, in the utility function definition (16), a relative weight $w_{k}=\frac{c_{k}}{T_{k}}$ is introduced to the gain or cost of link $k$,

\footnotetext{
${ }^{2}$ In this paper, a user is said having good (poor) channel quality if it is in an advantageous (disadvantageous) position, such as with a short (long) link distance and/or in a low (high)-interference neighborhood.
}

i.e.,

$$
U_{i}=\frac{c_{i}}{T_{i}} G_{i i}-\sum_{k \in \mathcal{S}} \frac{c_{k}}{T_{k}} C_{i k}
$$

where $c_{i}$ is the pre-specified priority factor (to represent the weight in resource sharing) of link $i$, and $T_{i}$ is the average throughput of link $i$ over a past window of length $t_{w}$. We use an exponential weighted low-pass filter to determine $T_{i}$,

$$
T_{i}=\left(1-\frac{1}{t_{w}}\right) T_{i}^{*}+\frac{1}{t_{w}} r_{i}
$$

where $T_{i}^{*}$ is the value of $T_{i}$ in the previous packet slot, and $r_{i}$ is the achieved transmission rate of link $i$ at the current packet slot.

From (18), it can be seen that, the better the channel quality of a link, the larger its gain in calculating the utility, thus obtaining higher priority in the scheduling. On the other hand, a link that does not transmit for a long time has an increased priority due to the smaller denominator in calculating its relative weight. It is shown in Section $\mathrm{V}$ that, using our proposed fairness adaptation, each link's service amount is approximately proportional to its channel quality.

As an example, Fig. 3 shows the detailed procedure of water-adding with fairness adaptation in a packet slot $l$. We omit the superscript of link activity $\alpha_{i}^{l}$. In the procedure, at the beginning of each packet slot, instead of randomly choosing a link to activate, the scheduler first activates the link with the largest relative weight.

\section{Fairness Index}

For UWB wireless networks, a fair resource allocation is not necessarily the case when each user receives the same service level. Consider a network with 3 links, where links 1 and 2 are close to each other while link 3 is far away from them. Links 1 and 2 generate large interference to each other. Hence, it is feasible not to allow them to be active simultaneously, i.e., the duty cycle of link 1 or 2 is at most $50 \%$. However, it is not good to require link 3 to have the same duty cycle as link 1 or 2 . As there is no large-interference link in link 3's neighborhood, it is better to allow link 3 to transmit all the time. This simple example shows that, to evaluate fairness, we should also take channel quality into account. However, it is challenging to evaluate the channel quality of a link in the UWB wireless network with peer-to-peer connections. It should be determined by the link's path loss and the interference level. Here we use a heuristic approach. For link $i$, define its interference set as the set of links that contribute/receive non-negligible interference to/from link $i$. We say link $i$ generates non-negligible interference to link $j$ 


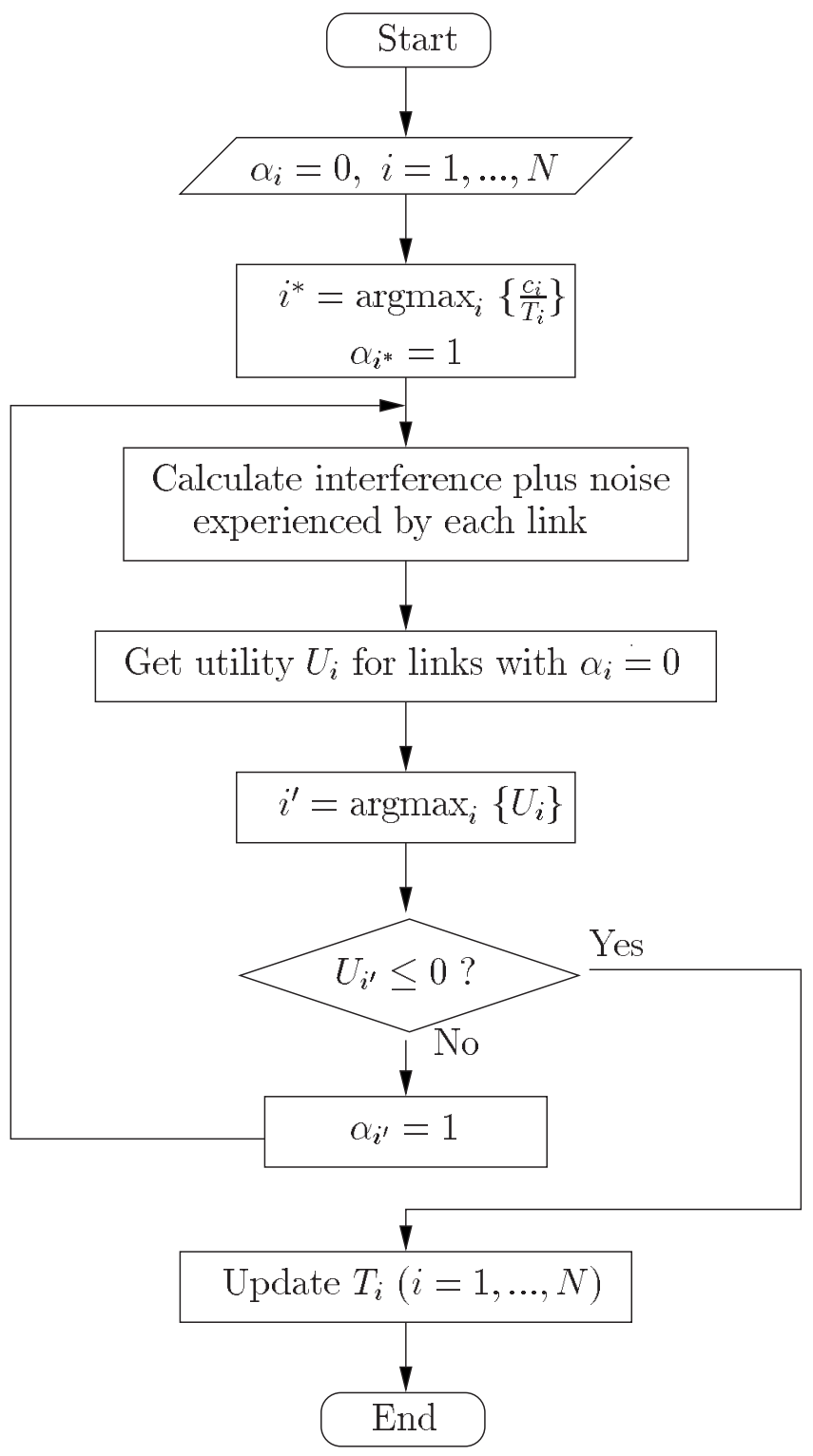

Fig. 3. The scheduling procedure (in a packet slot) of water-adding scheme with fairness adaptation.

if the normalized interference from link $i$ to $j$ (defined as the received interference from link $i$ 's transmitter normalized by the power of desired signal at link $j$ 's receiver) exceeds a threshold $F$. This is equivalent to $\frac{d_{i j}}{d_{j j}}<\beta$ when each active link transmits with the same maximum power, where parameter $\beta=F^{-1 / \theta}$ is termed the normalized distance threshold. Hence, for link $i$, its interference set can be denoted by

$$
I_{i}=\left\{j \mid j \neq i, \frac{d_{i j}}{d_{j j}}<\beta \text { or } \frac{d_{j i}}{d_{i i}}<\beta\right\}
$$

The size of set $I_{i}$ is denoted by $D\left(I_{i}\right)$. The value $q_{i}=$ $\frac{1}{1+D\left(I_{i}\right)}$ is an indication of channel quality. In general, the smaller the size of the interference set, the better the channel quality.

Based on the channel quality indicator, the fairness is measured by the Fairness Index [15] defined as

$$
\text { Fairness Index }=\frac{\left(\sum_{i=1}^{N} \frac{S_{i}}{c_{i} q_{i}}\right)^{2}}{N \cdot \sum_{i=1}^{N}\left(\frac{S_{i}}{c_{i} q_{i}}\right)^{2}}
$$

where $S_{i}$ is the average achieved rate of link $i$. The higher the Fairness Index value, the better the fairness performance. The upper bound of the Fairness Index is 1, which is achieved when $S_{i} / c_{i} q_{i}$ is independent of $i$.

\section{E. Adaptation to Long Acquisition Time}

One critical problem of UWB transmission is that the channel acquisition time can be quite long, which is a time to synchronize the receiver's clock with the transmitter's clock to achieve bit synchronization. Generally, for channel acquisition, the transmitter sends a preamble, whose duration usually varies from tens of microseconds to tens of milliseconds, as compared to microseconds in narrowband systems. This kind of overhead may greatly affect the bandwidth efficiency in high-speed UWB networks [16]. The relatively long acquisition time in UWB transmissions may also limit the UWB MAC design. Therefore, it is critical to design an efficient MAC protocol which keeps the effect of acquisition time as low as possible, in order to fully explore the high rate transmission.

For a UWB wireless network, if a link is scheduled to transmit in consecutive packet slots, the re-acquisition overhead for the packet slots following the first one can be avoided ${ }^{3}$. This means that it is desirable to let a link transmit continuously in terms of acquisition overhead reduction. To keep this in mind and, at the same time, to achieve acceptable system throughput and fairness performance, we modify the utility function in (18) to

$$
U_{i}=\frac{c_{i}}{T_{i}} \cdot(1-f(i) \cdot \xi) \cdot G_{i i}-\sum_{k \in \mathcal{S}} \frac{c_{k}}{T_{k}} \cdot(1-f(k) \cdot \xi) \cdot C_{i k}
$$

where

$$
f(i)= \begin{cases}0, & \text { for link } i \text { active at the previous packet slot } \\ 1, & \text { for link } i \text { idle at the previous packet slot }\end{cases}
$$

and $\xi$ is the acquisition overhead (defined as the portion of time in a packet slot for acquisition, if an acquisition is needed). The modified utility function favors transmission of a link at continuous packet slots.

\section{F. Further Discussion}

In this research, throughput and fairness are the main QoS consideration. This is our first-step study to the packet scheduling in UWB wireless networks. In order to implement our research in a more practical system, other important issues need further investigations, such as QoS in terms of delay and jitter, and stability of the scheduling schemes for a system with a finite buffer size. In general, these issues can be addressed in the following directions

\footnotetext{
${ }^{3}$ Although there exists a guard time between two packet slots, the short duration of the guard time is unlikely to destroy the bit synchronization between the sender and the receiver.
} 
TABLE I

THE SIMULATION PARAMETERS

\begin{tabular}{c|c||c|c}
\hline Symbol & Value & Symbol & Value \\
\hline \hline$P_{\max }$ & $0.5 \mathrm{~mW}$ & $\gamma$ & $10 \mathrm{~dB}$ \\
\hline$\eta$ & $4 \cdot 10^{-20} \mathrm{~W} / \mathrm{Hz}$ & $T_{f}$ & $100 \mathrm{~ns}$ \\
\hline$\sigma^{2}$ & $1.9966 \cdot 10^{-3}$ & $\theta$ & 2.4 \\
\hline$K$ & $1 / 1259$ & $t_{w}$ & 8 packet slots \\
\hline
\end{tabular}

- A call admission control is needed to limit the calls in service, so as to guarantee the QoS requirements of the admitted calls, and to make the system work in a stability region [17].

- Rate control in higher-layer protocols can help. For example, for video transmissions over UWB wireless networks, the source coding rate can adapt to the channel capacity. Further, with the bitplane coding, a fine granularity scalability (FGS) encoder [18] is capable of achieving a continuous rate as its enhancement bit stream can be truncated anywhere to achieve the target bit-rate. The sender may truncate the bit stream to fit the channel capacity to make the queue stable. On the other hand, for data transmission, the Transmission Control Protocol (TCP) can exploit the capacity on its path by adjusting its segment sending rate based on its congestion control mechanisms, thus making the system stable.

- To achieve stability and QoS (in terms of delay and jitter), the scheduler may need to incorporate the information of the packet delay experienced at each link [19] or the queue length of each link [20].

\section{Performance Evaluation}

The performance of the proposed resource allocation schemes are evaluated via computer simulations. We consider a UWB wireless network with $N=100$ long-lived links. Two test cases are simulated:

- Test case A: the 100 links are randomly located in a 10 $\mathrm{m} \times 10 \mathrm{~m}$ area.

- Test case B: the 100 links are randomly located in a 40 $\mathrm{m}$ x $40 \mathrm{~m}$ area.

In each test, the selected central controller collects the location information of each node, generates a map of the UWB network, and estimates the path gain of each link. The central controller is also responsible for the resource allocation of each packet slot. When active, link $i$ transmits with maximum power $P_{i}=P_{\max }$ and adapts transmission rate to the experienced interference with a required SINR value $\gamma$. We use simulation parameters similar to those in [11], [21], as listed in Table I. We only consider the overhead due to acquisition time for simplicity. For QoS provisioning, the bit-level QoS is guaranteed by adapting processing gain and/or channel coding to the experienced interference level. Hence, here we only investigate two packet-level QoS criteria: fairness and average achieved rate per link (which directly represents the system throughput).

\section{A. Comparison with All-at-Once and Exclusive Region Schemes}

Table II lists the Fairness Index, average achieved rate and normalized power consumption (with respect to $P_{\max }$ ) per link, and average number of active links per packet slot in All-at-Once (i.e., all links are active at all time), and in the proposed water-adding and water-draining schemes. In the simulation, the acquisition overhead $\xi$ and normalized distance threshold $\beta$ is set to 0 and 1 , respectively (the effects of different acquisition overhead and normalized distance threshold value are to be discussed in Sections V-C and V-B, respectively). All links are assigned the same priority factor $c_{i}=1$. It can be seen that, the average number of active links per packet slot in our schemes is around 37, a number large enough to validate the Gaussian approximation of the interference experienced by a link. Compared with the All-atOnce scheme, our proposed schemes in both tests significantly increase the Fairness Index from poor values (around 0.3 - 0.4) to acceptable ones (around 0.9); at the same time, the system throughput is also significantly increased, by approximately $30 \%$ and $70 \%$ in tests A and B, respectively. From the large Fairness Index values, it can be concluded that the achieved rate of each link is approximately proportional to its channel quality. In addition, the normalized power consumption per link in our proposed schemes is much less than that in the All-at-Once scheme, around $60 \%$ deduction in the above examples. It is preferable for UWB devices which normally have limited power supply and need to adhere to the strict emission regulation.

Comparisons are also carried out between our proposed schemes and the exclusive region scheme with a fixed exclusive region size (as used in [4], [5]). As it is not easy to select an appropriate exclusive region size, we test the exclusive region size ranging from $0-10$ meters in test $\mathrm{A}$, and $0-40$ meters in test B. To achieve a certain level of fairness, the exclusive region scheme operates as follows:

Step 1: All links are set as active.

Step 2: Set the target link (indexed by $i$ ) as the one with the largest relative weight $c_{i} / T_{i}$.

Step 3 : Mark the target link $i$ as "checked".

Step 4: Set idle the remaining active and un-checked links with transmitters located in the exclusive region of target link $i$.

Step 5: If all links are checked or idle, finish; otherwise, continue to Step 6.

Step 6 : From the remaining active and un-checked links, set the target link $i$ as the one with the largest relative weight, and continue to Step 3.

We also assume that each transmitter can adapt the processing gain and/or channel coding to the interference at the receiver so that the maximum achievable rate under the interference environment can be obtained. Note that when the exclusive region size is 0 , it is equivalent to the All-at-Once scheme, and when the exclusive region size is the size of the UWB wireless network, it is equivalent to Total-Exclusion scheme (i.e., only one link can transmit at any time).

In Figs. 4-7, we present the fairness and system throughput performance of the exclusive region scheme and our pro- 
TABLE II

FAIRNESS INDEX, AVERAGE ACHIEVED RATE AND NORMALIZED POWER CONSUMPTION PER LINK, AND AVERAGE NUMBER OF ACTIVE LINKS PER PACKET SLOT IN TESTS A AND B.

\begin{tabular}{c|c|c|c|c|c}
\hline Test & $\begin{array}{c}\text { Packet scheduling } \\
\text { scheme }\end{array}$ & $\begin{array}{c}\text { Fairness } \\
\text { Index }\end{array}$ & $\begin{array}{c}\text { Achieved rate } \\
\text { per link (Mbps) }\end{array}$ & $\begin{array}{c}\text { Normalized power } \\
\text { consumption per link }\end{array}$ & $\begin{array}{c}\text { Number of active } \\
\text { links per slot }\end{array}$ \\
\hline \hline & All-at-Once & 0.39 & 2.16 & 1 & 100 \\
\cline { 2 - 6 } A & Water-adding & 0.96 & 2.83 & 0.37 & 37.07 \\
\cline { 2 - 6 } & Water-draining & 0.91 & 2.92 & 0.37 & 37.23 \\
\hline \hline & All-at-Once & 0.33 & 1.61 & 1 & 100 \\
\cline { 2 - 6 } B & Water-adding & 0.96 & 2.76 & 0.37 & 36.75 \\
\cline { 2 - 6 } & Water-draining & 0.88 & 2.87 & 0.36 & 36.03 \\
\hline
\end{tabular}

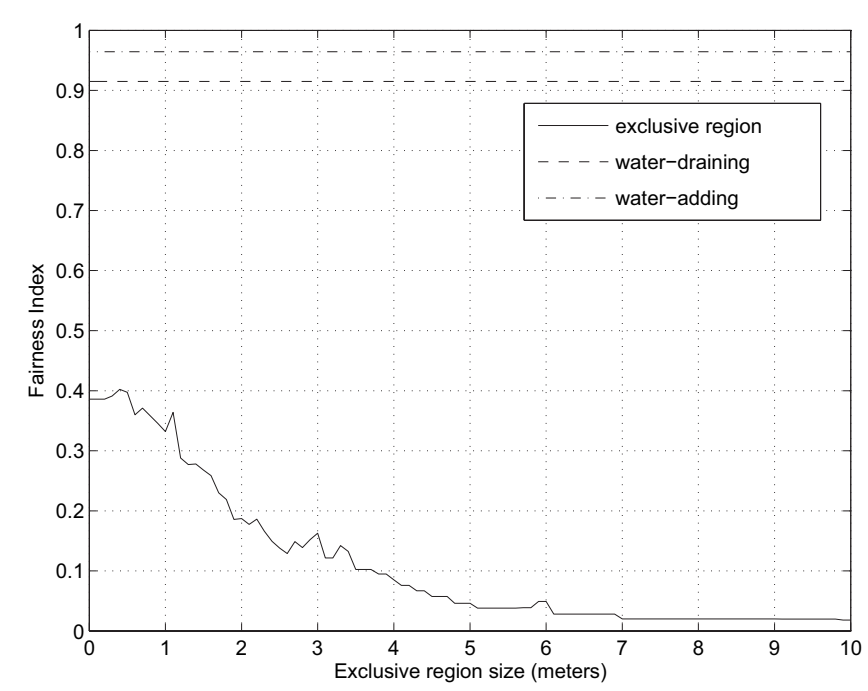

Fig. 4. Fairness Index of the exclusive region scheme and the proposed schemes in test A.

posed schemes in tests A and B. In both tests, the fairness performance of our proposed schemes is much better than that of the exclusive region scheme. In terms of system throughput, it can be seen that, for most exclusive region size values (except the neighborhood of 1 meter in test A), the achieved system throughput in our proposed schemes is much larger than that in the exclusive region scheme. The reason is that, different from the fixed-size exclusive region scheme, our schemes are dynamic ones, adapting to the different interference environment of each link. Next, we investigate the optimal exclusive region size in the exclusive region scheme. For test $\mathrm{A}$, it seems that the exclusive region should be set to around 1.1 meter. However, from Fig. 5, it is also observed that the system throughput is very sensitive to the exclusive region size around the optimal point, i.e., a small variation of the exclusive region size results in a large system throughput reduction. For test B, it is difficult to tell what is an appropriate exclusive region size, as there are several local maxima with a similar system throughput. The large fluctuations near the local maxima determine that it is not feasible to apply the exclusive region concept here. Furthermore, as the same maximum transmit power constraint is applied in both tests A and B, it can be concluded that the exclusive region should not depend only on the transmit power

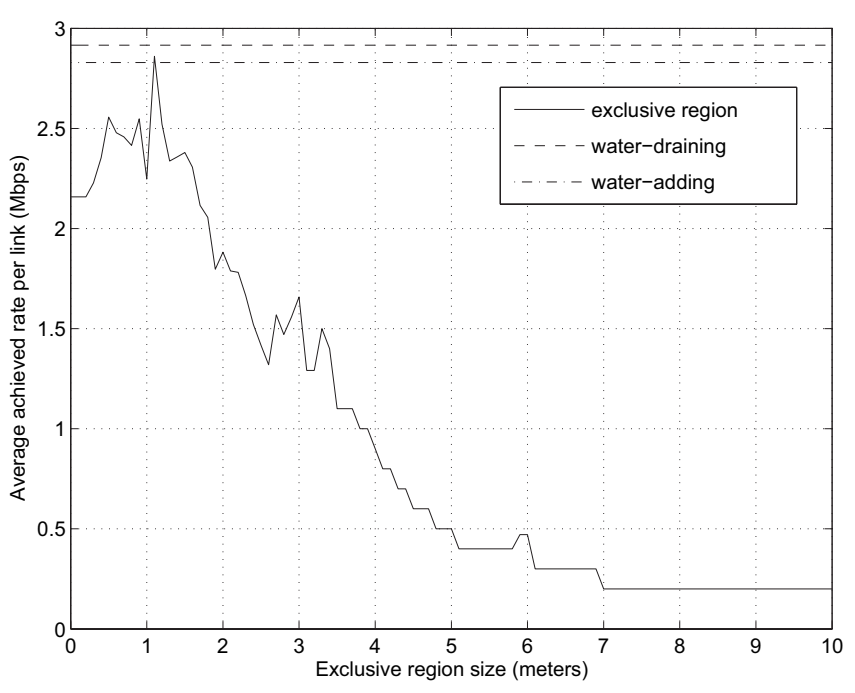

Fig. 5. Average achieved rate per link of the exclusive region scheme and the proposed schemes in test A.

constraint, which is different from the claim in [4] that the size of exclusive region depends only on the power constraint of the source.

\section{B. Effects of Normalized Distance Threshold $\beta$}

The normalized distance threshold $\beta$ is critical to evaluate the fairness performance of the proposed water-adding and water-draining schemes. Originally used to evaluate the channel quality of a link, $\beta$ should be set properly. Intuitively, it should not be very small, otherwise the estimated channel quality $q_{i}$ cannot properly indicate the actual situation of link $i$. In our simulation, we calculate the Fairness Index values in tests $\mathrm{A}$ and $\mathrm{B}$ for different $\beta$ values, shown in Fig. 8. It can be seen that, the Fairness Index value is not sensitive to $\beta$ when $\beta>0.3$. The calculated Fairness Index value decreases when $\beta$ decreases from 0.3 to 0.1 . This is because such small $\beta$ values cannot lead to a relatively accurate evaluation of the channel quality.

\section{Effects of Acquisition Time}

Simulations are carried out to check whether our proposed schemes can maintain efficiency with a relatively large acquisition overhead $\xi$. In the simulations, we ignore other 


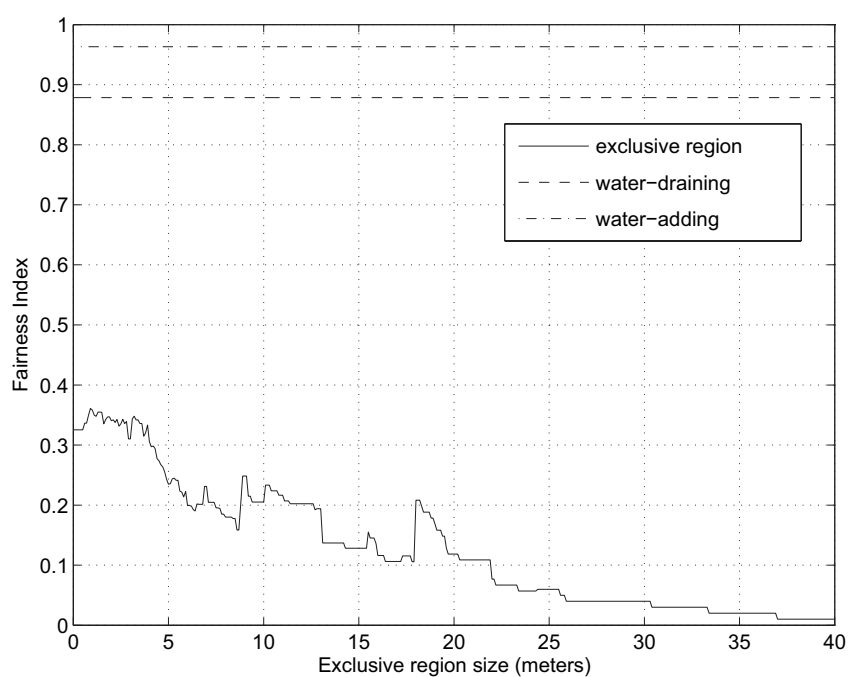

Fig. 6. Fairness Index of the exclusive region scheme and the proposed schemes in test B.

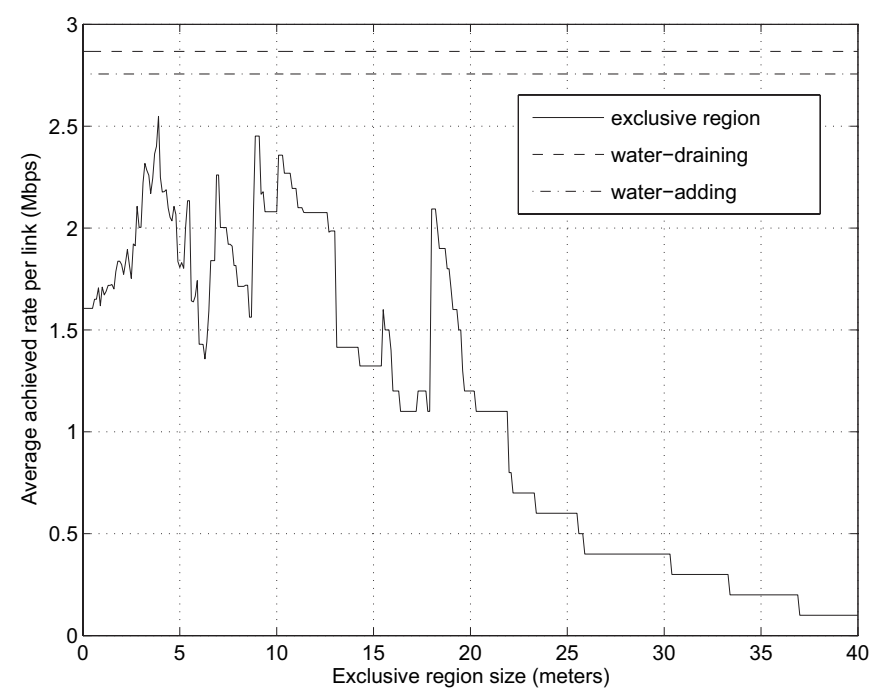

Fig. 7. Average achieved rate per link of the exclusive region scheme and the proposed schemes in test $\mathrm{B}$.

overheads and vary the acquisition overhead from 0 to 90 percent ${ }^{4}$. Figs. 9-11 show the average achieved rate per link, the Fairness Index, the average normalized power consumption (with respect to $P_{\max }$ ) per link, and the acquisition-necessary probability, respectively. Here the acquisition-necessary probability is defined as the probability that, when a link transmits at a packet slot, an acquisition is needed (i.e., the link is idle at the preceding packet slot). From Fig. 10, we can see that the average power consumption decreases slightly with the increase of acquisition overhead. This is because, when the acquisition overhead increases, the gain to include one link in a packet slot may not compensate for the loss of other links. Hence, the average active link number in a packet slot decreases accordingly, thus leading to a low average power consumption. From Fig. 9, it is interesting that, when the acquisition overhead increases from 0 to $90 \%$ (i.e., the system efficiency significantly decreases from $100 \%$ to

\footnotetext{
${ }^{4}$ The 90 percent may be an extreme case. We use it here to demonstrate the performance of our proposed schemes under severe conditions.
}

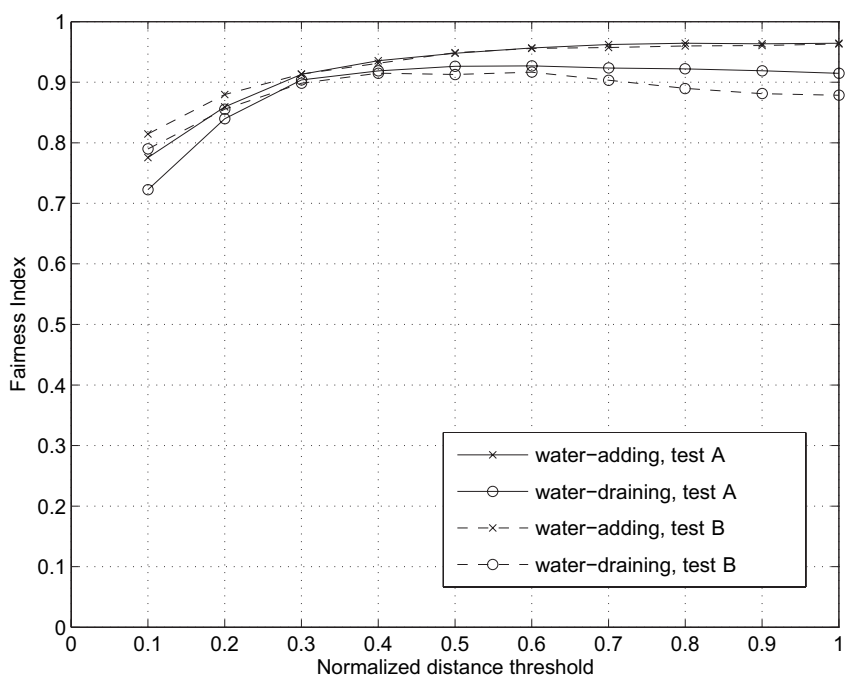

Fig. 8. Fairness Index versus normalized distance threshold $\beta$.

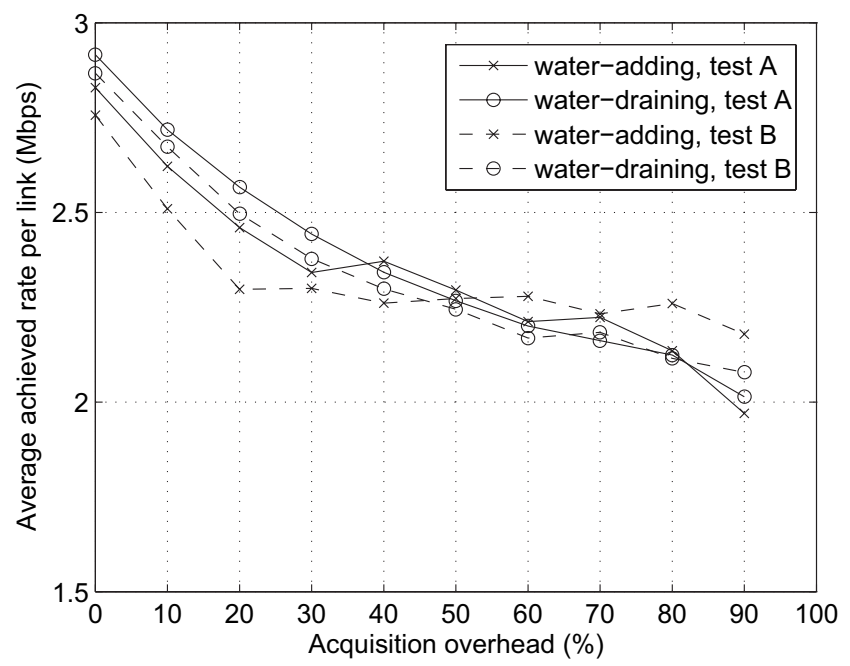

Fig. 9. Average achieved rate versus acquisition overhead $\xi$.

$10 \%$ when an acquisition is needed), the average achieved rate per link only decreases by approximately $30 \%$. This is because, with a large acquisition overhead, our proposed schemes can automatically favor transmission of a link at consecutive packet slots. This can also be seen from Fig. 11 , where the acquisition-necessary probability decreases from values around 0.8 (at the acquisition overhead equal to 0 ) to approximately 0.2 (at the acquisition overhead equal to $90 \%$ ). In addition, the fairness performance of our proposed schemes is not affected by different acquisition overhead values, as shown in Fig. 10. The simulation results demonstrate that our proposed schemes are robust to the relatively long acquisition time in UWB networks.

\section{Effects of Shadowing}

In this research, a UWB network with low mobility is considered. We assume that there is no fast fading, due to the rich resolvable multipath components in UWB transmissions. However, it is not easy to compensate for signal attenuation due to shadowing. In this subsection, we evaluate the effect 


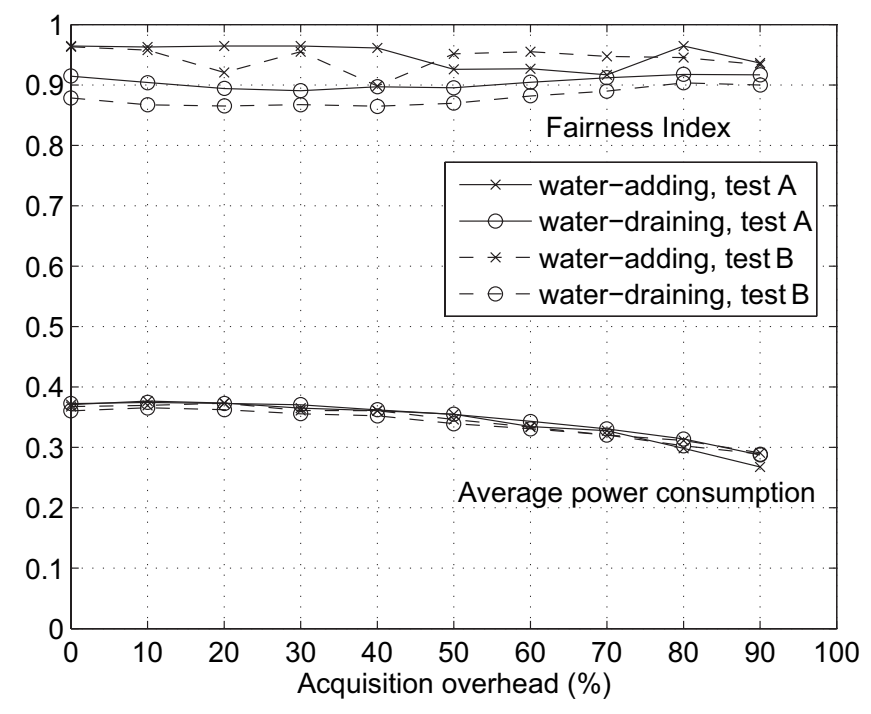

Fig. 10. Fairness Index and average normalized power consumption (with respect to $P_{\max }$ ) per link versus acquisition overhead $\xi$.

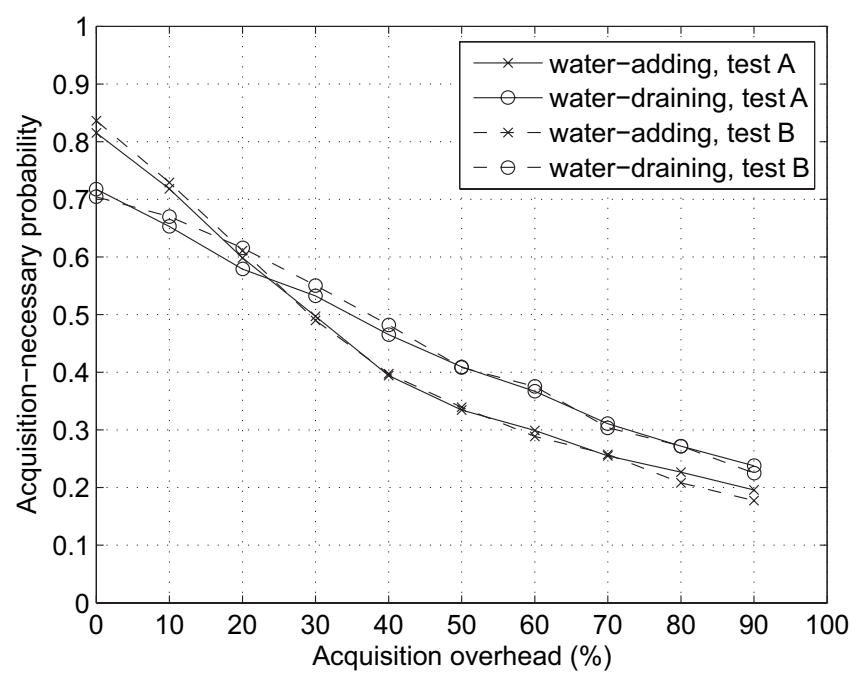

Fig. 11. Acquisition-necessary probability versus acquisition overhead $\xi$.

of shadowing on throughput and fairness performance of the proposed packet scheduling schemes.

When lognormal shadowing is considered, the channel gain from link $i$ 's transmitter to link $j$ 's receiver in (1) should be rewritten as

$$
h_{i j}=K \cdot d_{i j}^{-\theta} \cdot 10^{\chi_{i j} / 10}
$$

where $\chi_{i j}$ (the $\mathrm{dB}$ attenuation due to shadowing) is a zeromean Gaussian random variable with variance $\sigma_{\chi}^{2}$. To the best of our knowledge, so far there is no research result in the open literature on correlation property of UWB channel shadowing. At a first step, we use the first-order autoregressive process [22] (used for a narrowband channel) to model UWB channel shadowing. Let $\chi_{i j}(l)$ denote the $\chi_{i j}$ value at the $l$ th packet slot. The random process $\chi_{i j}(l)$ can be modeled by

$$
\chi_{i j}(l)=\zeta^{v \cdot t_{\text {slot }}} \cdot \chi_{i j}(l-1)+\left(1-\zeta^{v \cdot t_{\text {slot }}}\right) \cdot n(l)
$$

where $\zeta$ is the correlation between two points separated by one meter, $v$ is the moving velocity, $t_{\text {slot }}$ is the duration of a packet slot, and $n(l)$ 's for different $l$ values are i.i.d. Gaussian random variables with zero mean and standard deviation $\sigma_{\chi} \sqrt{\frac{1+\zeta^{v \cdot t_{\text {slot }}}}{1-\zeta^{v \cdot t_{\text {slot }}}}}$.

In the system, each transmitter/receiver pair adjusts the transmission rate according to the received desired signal power and interference level. The transmitter/receiver pair informs the central controller their location, the channel gain, interference level, and actually received service amount after every $M$ packet slots. The value of $M$ is determined experimentally.

We set $\xi=10 \%, \beta=1, \sigma_{\chi}=4 \mathrm{~dB}, \zeta=0.9, t_{\text {slot }}=5 \mathrm{~ms}$ in the simulation. Test case B with the water-adding scheme is used as an example. The velocity $v$ varies from $0.2 \mathrm{~m} / \mathrm{s}, 2 \mathrm{~m} / \mathrm{s}$, to $10 \mathrm{~m} / \mathrm{s}^{5}$. All the senders are moving with velocity $v$, while the receivers are static. Each sender chooses a new moving direction randomly in $[0,2 \pi)$ at the end of each second. Table III shows the average achieved rate per link and fairness performance with different $v$ and $M$ value in test $\mathrm{B}$ with the water-adding scheme. For comparison, the performance with no mobility/shadowing is also given. When $v=0.2 \mathrm{~m} / \mathrm{s}$, the throughput and fairness performance with mobility/shadowing is degraded slightly from that with no mobility/shadowing. This is because of the very low variance in shadowing. It is also observed that the throughput and fairness performance is not very sensitive to the selection of the $M$ value. Thus, a relative large $M$ value (e.g., 1000) can be used. When $v=2$ $\mathrm{m} / \mathrm{s}$, we can see that it is better to select $M=200$. For the extreme case $v=10 \mathrm{~m} / \mathrm{s}, M=50$ is appropriate.

\section{E. Implementation and Discussion}

Our proposed schemes dynamically allocate resources to the transmission links according to their channel quality and average received services. They can achieve desired QoS requirements at the cost of certain computation complexity at each packet slot. However, the complexity of our proposed schemes can be reduced in a practical system. For the resource allocation, time can be partitioned into cycles. Each cycle consists of one or several frames, as long as the number of packet slots in a cycle is large enough to achieve the required fairness level. The resource allocation remains the same from cycle to cycle, until the central controller announces a new resource allocation decision due to call arrivals/departures or user mobility. When the central controller detects a significant distance update of the users, it re-allocates the resources for each cycle, and broadcasts the allocation results to the users. With low mobility, the re-allocation frequency is not high, thus not leading to a large computation burden to the central controller.

\section{CONCLUSIONS}

For UWB wireless networks, the exclusive region for specific links should be a system-level concept, taking into account interference from/to other existing active links. From the point of view, we propose the water-draining and wateradding scheduling schemes to achieve a good compromise

\footnotetext{
${ }^{5}$ The $10 \mathrm{~m} / \mathrm{s}$ velocity may be too large for a low-mobility UWB wireless network in a small area. We use it here to demonstrate the performance of our proposed schemes under extreme conditions.
} 
TABLE III

THE AVERAGE ACHIEVED RATE PER LINK AND FAIRNESS INDEX IN TEST B COMPARED WITH NO-MOBILITY/SHADOWING CASE IN THE WATER-ADDING SCHEME.

\begin{tabular}{c|c|c|c|c|c|c|c|c}
\hline \multicolumn{2}{c|}{$M$} & 1 & 10 & 50 & 100 & 200 & 1000 & No-mobility/shadowing \\
\hline \hline \multirow{4}{*}{$\begin{array}{c}\text { Rate } \\
(\mathrm{Mbps})\end{array}$} & $v=0.2 \mathrm{~m} / \mathrm{s}$ & 2.3754 & 2.3705 & 2.3692 & 2.3781 & 2.3716 & 2.3865 & \multirow{2}{*}{2.4569} \\
\cline { 2 - 8 } & $v=2 \mathrm{~m} / \mathrm{s}$ & 2.0342 & 1.9827 & 2.0099 & 1.9814 & 1.9705 & 1.8770 & \\
\cline { 2 - 8 } & $v=10 \mathrm{~m} / \mathrm{s}$ & 1.9531 & 1.9806 & 1.9508 & 1.8561 & 1.7620 & 1.3693 & \\
\hline \multirow{2}{*}{$\begin{array}{c}\text { Fairness } \\
\text { Index }\end{array}$} & $v=0.2 \mathrm{~m} / \mathrm{s}$ & 0.8831 & 0.8891 & 0.8913 & 0.8870 & 0.8881 & 0.8798 & \multirow{2}{*}{0.9386} \\
\cline { 2 - 7 } & $v=2 \mathrm{~m} / \mathrm{s}$ & 0.8738 & 0.9110 & 0.8929 & 0.8849 & 0.9111 & 0.8546 & \\
\cline { 2 - 6 } & $v=10 \mathrm{~m} / \mathrm{s}$ & 0.9323 & 0.9162 & 0.9442 & 0.9124 & 0.9224 & 0.7583 & \\
\hline \hline
\end{tabular}

between throughput and fairness. Each link's achieved rate is approximately proportional to its channel quality level. Via computer simulations, we have shown that our proposed schemes outperform the All-at-Once scheme and the exclusive region scheme with a fixed exclusive region size. To develop an effective and efficient MAC for UWB wireless networks, further research efforts are necessary in call admission control with QoS guarantees, resource allocation with delay and stability consideration, and cross-layer design between the link layer packet scheduling and high-layer rate control mechanisms, etc.

\section{REFERENCES}

[1] W. Zhuang, X. Shen, and Q. Bi, "Ultra-wideband wireless communications," Wireless Commun. and Mobile Comput., vol. 3, no. 6, pp. 663-685, Sept. 2003.

[2] R. Negi and A. Rajeswaran, "Capacity of power constrained ad-hoc networks," in Proc. IEEE INFOCOM'04, pp. 443-453.

[3] R. Negi and A. Rajeswaran, "Scheduling and power adaptation for networks in the ultra-wideband regime," in Proc. IEEE GLOBECOM'04, pp. 139-145.

[4] B. Radunovic and J.-Y. Le Boudec, "Optimal power control, scheduling, and routing in UWB networks," IEEE J. Sel. Areas Commun., vol. 22, no. 7, pp. 1252-1270, Sept. 2004.

[5] J.-Y. Le Boudec, R. Merz, B. Radunovic, and J. Widmer, "A MAC protocol for UWB very low power mobile ad hoc networks based on dynamic channel coding with interference mitigation," EPFL-DI-ICA, Tech. Rep. IC/2004/02, Jan. 2004.

[6] F. Ramirez-Mireles, "On the performance of ultra-wide-band signals in Gaussian noise and dense multipath," IEEE Trans. Veh. Technol., vol. 50, no. 1, pp. 244-249, Jan. 2001

[7] K. W. Lam, Q. Li, L. Tsang, K. L. Lai, and C. H. Chan, "On the analysis of statistical distributions of UWB signal scattering by random rough surfaces based on Monte Carlo simulations of Maxwell equations," IEEE Trans. Antenna Propagat., vol. 52, no. 12, pp. 3200-3206, Dec. 2004.

[8] M. Z. Win and R. A. Scholtz, "On the robustness of ultra-wide bandwidth signals in dense multipath environments," IEEE Commun. Lett., vol. 2, no. 2, pp. 51-53, Feb. 1998 .

[9] IEEE P802.15-03/268r3, Multi-Band OFDM Physical Layer Proposal for IEEE 802.15 Task Group 3a, Mar. 2004. Project: IEEE P802.15 Working Group for Wireless Personal Area Networks (WPANs).

[10] M. Z. Win and R. A.Scholtz, "Ultra-wide bandwidth time-hopping spread-spectrum impulse radio for wireless multiple-access communications," IEEE Trans. Commun., vol. 48, no. 4, pp. 679-691, Apr. 2000.

[11] F. Cuomo, C. Martello, A. Baiocchi, and F. Capriotti, "Radio resource sharing for ad hoc networking with UWB," IEEE J. Sel. Areas Commun., vol. 20, no. 9, pp. 1722-1732, Dec. 2002

[12] S. Toumpis and A. J. Goldsmith, "Capacity regions for wireless ad hoc networks," IEEE Trans. Wireless Commun., vol. 2, no. 4, pp. 736-748, July 2003.

[13] H. Luo, J. Cheng, and S. Lu, "Self-coordinating localized fair queueing in wireless ad hoc networks," IEEE Trans. Mobile Computing, vol. 3, no.1, pp. 86-98, Jan.-Mar. 2004.
[14] J. M. Holtzman, "Asymptotic analysis of proportional fair algorithm," in Proc. IEEE PIMRC'01, pp. F-33-F-37.

[15] R. Jain, A. Durresi, and G. Babic, "Throughput fairness index: an explanation," ATM Forum Document Number: ATM_Forum/99-0045, Feb. 1999.

[16] S. Roy, J. R. Foerster, V. S. Somayazulu, and D. G. Leeper, "Ultrawideband radio design: the promise of high-speed, short-range wireless connectivity," Proc. of the IEEE, vol. 92, no. 2, pp. 295-311, Feb. 2004.

[17] L. Tassiulas and A. Ephremides, "Stability properties of constrained queueing systems and scheduling policies for maximum throughput in multihop radio networks," IEEE Trans. Automatic Control, vol. 37, no. 12, pp. 1936-1948, Dec. 1992.

[18] W. Li, "Overview of fine granularity scalability in MPEG-4 video standard," IEEE Trans. Circuits Syst. Video Technol., vol. 11, no. 3, pp. 301-317, Mar. 2001.

[19] M. Andrews, K. Kumaran, K. Ramanan, A. Stolyar, P. Whiting, and R. Vijayakumar, "Providing quality of service over a shared wireless link," IEEE Commun. Mag., vol. 39, no. 2, pp. 150-154, Feb. 2001.

[20] A. Eryilmaz, R. Srikant, and J. R. Perkins, "Stable scheduling policies for fading wireless channels," IEEE/ACM Trans. Networking, vol. 13, no. 2, pp. 411-424, Apr. 2005

[21] F. Cuomo, C. Martello, and S. Caputo, "An interference-controlled admission control scheme for QoS support in distributed UWB networks", in Proc. IST Mobile Communications Summit 2003, pp. 508-512.

[22] G. L. Stüber, Principles of Mobile Communication. Kluwer Academic Publishers, 1996, pp. 89-90.

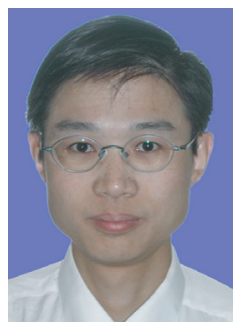

Hai Jiang (S'04) received the B.S. degree in 1995 and the M.S. degree in 1998, both in electronics engineering, from Peking University, Beijing, China, and the $\mathrm{Ph}$.D. degree in 2006 in electrical engineering from the University of Waterloo, Canada. He is currently a Postdoctoral Fellow at the Department of Electrical Engineering, Princeton University. His research interests include radio resource management, cellular/WLAN interworking, and cross-layer design for wireless multimedia communications.

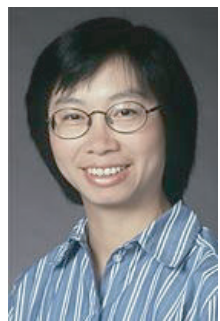

Weihua Zhuang (M'93-SM'01) has been a Professor in the Department of Electrical and Computer Engineering, University of Waterloo, Canada, since 1993. Dr. Zhuang is a co-author of the textbook Wireless Communications and Networking (Prentice Hall, 2003). Her current research interests include multimedia wireless communications, wireless networks, and radio positioning. She received the Outstanding Performance Award in 2005 from the University of Waterloo and the Premier's Research Excellence Award (PREA) in 2001 from the Ontario Government. She is an Editor/Associate Editor of IEEE Transactions on Wireless Communications, IEEE Transactions on Vehicular Technology, and EURASIP Journal on Wireless Communications and Networking. 